\title{
Synthesis and structural characterization of new inorganic-organic hybrid: arsenomolybdate compound with cytosinium cations
}

\author{
MERIEM AYED*, BRAHIM AYED and AMOR HADDAD \\ Laboratoire des matériaux et cristallochimie (LMC), Faculté des Sciences de Monastir, 5000 Monastir, Tunisia
}

MS received 18 January 2013; revised 21 March 2013

\begin{abstract}
New organic-inorganic hybrid compound, with formula $\left(\mathrm{C}_{4} \mathrm{H}_{6} \mathrm{~N}_{3} \mathrm{O}\right)_{6}\left[\left(\mathrm{HAsO}_{4}\right)_{2} \mathrm{Mo}_{6} \mathrm{O}_{19}\right] \cdot 7 \mathrm{H}_{2} \mathrm{O}$, was prepared and characterized by IR and UV-visible spectroscopies and X-ray diffraction techniques. Thermal analysis was performed to study their thermal stability. The crystal structure of the title compound (triclinic, space group $P-1, Z=2)$ was determined by $\mathrm{X}$-ray diffraction. The compound contains the polyanion $\left[\left(\mathrm{HAsO}_{4}\right)_{2} \mathrm{Mo}_{6} \mathrm{O}_{19}\right]^{6-}$, which consists of the six molybdenum octahedral grouped into two parts consisting of four edge-sharing octahedral and two face-sharing octahedral, respectively, these two parts are connected by two corner-sharing $\mathrm{O}$ atoms to form a bent $\mathrm{Mo}_{6}$ ring. The polyanion framework derives from the Strandberg type and it is a new isomer. The cytosinium cations $\left(\mathrm{Cyt}^{+}\right)$are embedded in the channels and interact with the inorganic framework by way of $\mathrm{N}-\mathrm{H} \cdot \cdots \mathrm{O}$ and $\mathrm{O}-\mathrm{H} \cdots \mathrm{O}$ hydrogen bonds. Furthermore, the electrochemical property of this compound has been studied.
\end{abstract}

Keywords. Arsenomolybdate; crystal structure; thermal behaviour; infrared spectroscopy; cytosine.

\section{Introduction}

Polyoxometalates (POMs) have been a centre of research interest for a long time, as they exhibit a great variety of structures and rich diversity of remarkable properties. ${ }^{1-7}$ In the past decades, an important advance in polyoxometalate chemistry is the polyoxometalate-based organic-inorganic hybrid materials. ${ }^{8,9}$ Such materials not only demonstrate their fascinating structural diversities, but also provide new and exciting functional materials with interesting optical, electronic and magnetic or catalytic properties. ${ }^{10-12}$ In particular, polyoxomolybdates shows a great variety of structures and properties. A large number of compounds were synthesized and structurally characterized in the $A-\mathrm{Mo}-\mathrm{P}-\mathrm{O}$ system, where $A$ is an organic or inorganic cation. ${ }^{13-15}$ They exhibit varieties of novel frameworks. In contrast to the rich structural chemistry of molybdenum phosphates, the Mo-As-O system remains relatively undeveloped. In recent years, chemists have obtained some compounds of Mo-As-O system belonging to a new class of anion clusters distinctly different from Keggin ion $\left[\mathrm{AsMo}_{12} \mathrm{O}_{40}\right]^{3-}$ and Dawson polyanion $\left[\mathrm{As}_{2} \mathrm{Mo}_{18} \mathrm{O}_{62}\right]^{6-}$, such as $\left[\mathrm{As}_{6} \mathrm{Mo}_{4} \mathrm{O}_{22} \mathrm{H}_{2}\right]^{4-},{ }^{16,17}$ $\left[\mathrm{AsMo}_{8} \mathrm{O}_{30}\right]^{7-},{ }^{18}\left[\left(\mathrm{HAsO}_{4}\right)_{2}\left(\mathrm{Mo}_{2} \mathrm{O}_{5}\right)\right]^{2-19}$ and $\left[\mathrm{As}_{3} \mathrm{Mo}_{12}\right.$ $\left.\mathrm{O}_{40}\right]^{5-} \cdot 16,17$ In order to obtain new materials with novel structures and interesting properties, we report here the synthesis and X-ray crystal structure of a novel inorganicorganic hybrid arsenomolybdate compound with cytosinium cations.

\footnotetext{
*Author for correspondence (meriem.ayed@hotmail.fr)
}

\section{Experimental}

\subsection{Materials and measurements}

All chemicals were purchased commercially and used without any further purification. Qualitative analysis by scanning electron microscopy (SEM) probe was performed using an apparatus of the type JOEL JSM-5400 (JOEL Ltd Tokyo, Japan). Infrared spectrum was recorded at room temperature on a Nicolet 470 FTIR spectrophotometer as KBr pellets in the $4000-400 \mathrm{~cm}^{-1}$ region. UV-vis spectrum was measured using a Perkin-Elmer Lambda 19 spectrophotometer in the 180-800 $\mathrm{nm}$ range. The TGA-DTA thermograms were obtained with $3.5 \mathrm{mg}$. Sample was placed in an open platinum crucible and heated, under air, from room temperature to $450^{\circ} \mathrm{C}$ with $5^{\circ} \mathrm{C} \mathrm{min}^{-1}$ heating rate; an empty crucible was used as reference. A CHI 660 Electrochemical Workstation connected to a Digital-586 personal computer was used for control of the electrochemical measurements. A conventional three-electrode system was used. The working electrode was gold, platinum gauze was used as counter electrode and the saturated calomel electrode (SCE) was the reference electrode. The measurements were performed at room temperature.

\subsection{Synthesis}

A solution of cytosine base $\mathrm{C}_{4} \mathrm{H}_{5} \mathrm{~N}_{3} \mathrm{O}(0.5 \mathrm{mmol})$ in 10 $\mathrm{cm}^{3}$ of water was added, with vigorous stirring, to a mixture of $\mathrm{Na}_{2} \mathrm{MoO}_{4} \cdot 2 \mathrm{H}_{2} \mathrm{O}(1 \mathrm{mmol})$ and $\mathrm{H}_{3} \mathrm{AsO}_{4}(3 \mathrm{mmol})$ in $20 \mathrm{~cm}^{3}$ of water, at room temperature with a starting $\mathrm{pH} 3$ adjusted with glacial acetic acid. One week later, colourless 


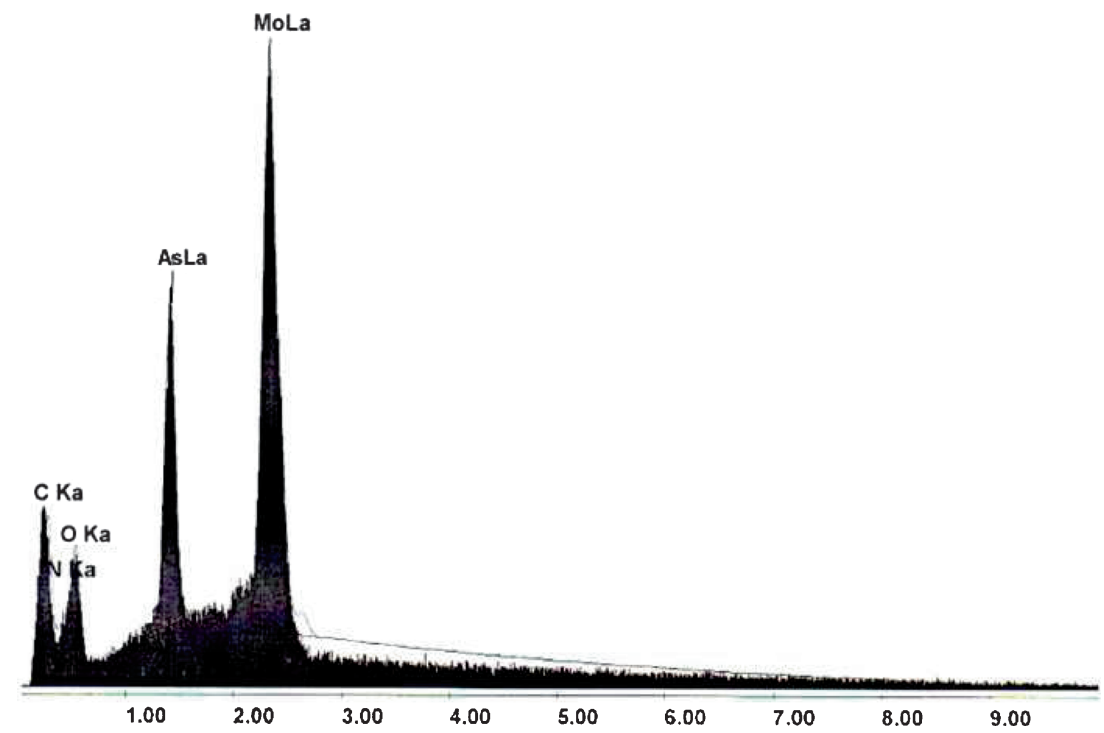

Figure 1. SEM spectrum of $\left(\mathrm{C}_{4} \mathrm{H}_{6} \mathrm{~N}_{3} \mathrm{O}\right)_{6}\left[\left(\mathrm{HAsO}_{4}\right)_{2} \mathrm{Mo}_{6} \mathrm{O}_{19}\right] \cdot 7 \mathrm{H}_{2} \mathrm{O}$.

block crystals were grown by slow evaporation at room temperature. The qualitative analysis by SEM of one of the colourless stick-like crystals obtained revealed the presence of only Mo, As, C, N and $\mathrm{O}$ elements (figure 1). Elem. Anal. Found: Mo, 29.4; As, 7.65; C, 14.72; N, 12.87; O, 32.68 (\%). Calcd: Mo, 29.7; As, 7.62; C, 14.58; N, 13.10; O, $32.68(\%)$.

\section{Results and discussion}

\subsection{X-ray crystallographic study}

Single crystal of the title compound with dimension $0.1 \times$ $0.2 \times 0.15 \mathrm{~mm}^{3}$ was chosen for X-ray diffraction studies. Data were collected on an Enraf-Nonius CAD-4 diffractometer with $\mathrm{MoK}_{\alpha}$ monochromatic radiation $(\lambda=0.71073 \AA)^{20}$ at $293 \mathrm{~K}$. Empirical absorption correction was applied using $\psi$-scan data. ${ }^{21}$ The structure was successfully developed in the non-centrosymmetric space group $P-1$, solved by the Patterson method using SHELXS-97 22,23 and refined by the full-matrix least-squares method on $F^{2}$ using the SHELXL-97 crystallographic software package. $^{22,23}$

A total of 12460 reflections $\left(2.088^{\circ}<\theta<26.978^{\circ}\right)$ was collected, of which 12460 unique reflections $[R$ (int $)=0.0282$ ] were used. The structure was solved using SHELXS-97 and refined using SHELXL-97 to $R_{1}=0.060, \mathrm{w} R_{2}=$ 0.141 , for 9627 reflections with $I>2 \sigma(I)$. All non-hydrogen atoms were refined anisotropically. Attempt to locate the positions of some hydrogen atoms from different Fourier maps was given up. Further details of the X-ray structural analysis are given in table 1 . The final atomic coordinates and equivalent temperature factors are presented in table 2. Selected bond lengths of 1 are listed in table 3 . Structure graphics are drawn with DIAMOND 2.1. ${ }^{36}$
Table 1. Crystal data and structure refinement for $\left(\mathrm{C}_{4} \mathrm{H}_{6} \mathrm{~N}_{3} \mathrm{O}\right)_{6}$ $\left[\left(\mathrm{HAsO}_{4}\right)_{2} \mathrm{Mo}_{6} \mathrm{O}_{19}\right] \cdot 7 \mathrm{H}_{2} \mathrm{O}$.

\begin{tabular}{ll}
\hline Chemical formula & $\left(\mathrm{C}_{4} \mathrm{H}_{6} \mathrm{~N}_{3} \mathrm{O}\right)_{6}\left[\left(\mathrm{HAsO}_{4}\right)_{2} \mathrm{Mo}_{6} \mathrm{O}_{19}\right] \cdot 7 \mathrm{H}_{2} \mathrm{O}$ \\
Formula weight & $1956.14 \mathrm{~g} \mathrm{~mol}^{-1}$ \\
Crystal system & Triclinic \\
Space group & $P-1$ \\
$a(\AA)$ & $11.0334(1)$ \\
$b(\AA)$ & $15.3654(1)$ \\
$c(\AA)$ & $18.3757(1)$ \\
$\alpha\left({ }^{\circ}\right)$ & $101.710(1)$ \\
$\beta\left({ }^{\circ}\right)$ & $106.889(1)$ \\
$\gamma\left(\left(^{\circ}\right)\right.$ & $92.986(1)$ \\
$V\left(\AA^{3}\right)$ & $2898.30(4)$ \\
$Z$ & 2 \\
$T(\mathrm{~K})$ & $293(2)$ \\
$\mu_{\mathrm{Mo}(\mathrm{K} \alpha)}\left(\mathrm{mm}^{-1}\right)$ & 2.445 \\
$R_{1}[I>2 \sigma(I)]$ & 0.033 \\
$\mathrm{w} R_{2}$ & 0.087 \\
\hline
\end{tabular}

\subsection{Structure description}

Single-crystal $\mathrm{X}$-ray structural analysis shows that the structure of $\left(\mathrm{C}_{4} \mathrm{H}_{6} \mathrm{~N}_{3} \mathrm{O}\right)_{6}\left[\left(\mathrm{HAsO}_{4}\right)_{2} \mathrm{Mo}_{6} \mathrm{O}_{19}\right] \cdot 7 \mathrm{H}_{2} \mathrm{O}$ consists of isolated $\left[\left(\mathrm{HAsO}_{4}\right)_{2} \mathrm{Mo}_{6} \mathrm{O}_{19}\right]^{6-}$ cluster anions, protonated cytosine $\left(\mathrm{C}_{4} \mathrm{H}_{6} \mathrm{~N}_{3} \mathrm{O}\right)^{+}$and water molecules. A projection of the structure, showing the displacement ellipsoids, is presented in figure 2. The $\left\{\mathrm{As}_{2} \mathrm{Mo}_{6}\right\}$ anion is formed by close packing of oxygen atoms with As and Mo atoms in the distorted tetrahedral and octahedral voids, respectively. A perspective view of the $\left[\left(\mathrm{HAsO}_{4}\right)_{2} \mathrm{Mo}_{6} \mathrm{O}_{19}\right]^{6-}$ anion with its numbering scheme is shown in figure 3 . The polyoxomolybdate cluster $\left[\left(\mathrm{HAsO}_{4}\right)_{2} \mathrm{Mo}_{6} \mathrm{O}_{19}\right]^{6-}$ can be described as a ring of six distorted edge-, face- and corner-sharing $\mathrm{MoO}_{6}$ octahedra with two $\mathrm{HAsO}_{4}$ tetrahedra capped on each side. The arsenic As2 subunit shares three oxo-groups 
Table 2. The final atomic coordinates, fractional occupancies and equivalent temperature factors.

\begin{tabular}{|c|c|c|c|c|c|}
\hline Atoms & $x$ & $y$ & $z$ & Occupation & $U_{\text {éq }}$ \\
\hline As 1 & $0.89840(4)$ & $0.37987(3)$ & $0.13234(2)$ & 1 & $0.01417(9)$ \\
\hline As 2 & $0.78464(4)$ & $0.35147(3)$ & $0.30854(3)$ & 1 & $0.01844(10)$ \\
\hline Mo1 & $1.09704(4)$ & $0.31269(2)$ & $0.31203(2)$ & 1 & $0.02039(9)$ \\
\hline Mo2 & $0.71558(3)$ & $0.16253(2)$ & $0.02990(2)$ & 1 & $0.01820(9)$ \\
\hline Mo3 & $1.01929(4)$ & $0.52302(2)$ & $0.31915(2)$ & 1 & $0.01973(9)$ \\
\hline Mo4 & $1.00448(4)$ & $0.15343(2)$ & $0.11900(2)$ & 1 & $0.02223(9)$ \\
\hline Mo5 & $0.72134(4)$ & $0.53518(2)$ & $0.20821(2)$ & 1 & $0.02023(9)$ \\
\hline Mo6 & $0.55701(3)$ & $0.33460(2)$ & $0.12441(2)$ & 1 & $0.01908(9)$ \\
\hline $\mathrm{O} 1$ & $0.3206(6)$ & $0.7690(5)$ & $0.1488(6)$ & 1 & $0.0277(14)$ \\
\hline $\mathrm{O} 2$ & $0.2995(7)$ & $0.3333(5)$ & $0.3595(6)$ & 1 & $0.0338(15)$ \\
\hline $\mathrm{O} 3$ & $0.3837(8)$ & $0.4967(8)$ & $0.3816(8)$ & 1 & $0.021(2)$ \\
\hline $\mathrm{O} 4$ & $0.4855(11)$ & $0.6391(8)$ & $0.3018(10)$ & 1 & $0.025(2)$ \\
\hline O5 & $0.4080(8)$ & $0.4723(7)$ & $0.1603(8)$ & 1 & $0.016(2)$ \\
\hline O6 & $0.6442(8)$ & $0.5120(9)$ & $0.4841(7)$ & 1 & $0.020(2)$ \\
\hline $\mathrm{O} 7$ & $0.6614(7)$ & $0.4993(7)$ & $0.2814(7)$ & 1 & $0.0159(18)$ \\
\hline O8 & $0.5184(10)$ & $0.3498(8)$ & $0.3570(9)$ & 1 & $0.021(2)$ \\
\hline O9 & $0.8525(10)$ & $0.6076(8)$ & $0.4851(10)$ & 1 & $0.026(2)$ \\
\hline $\mathrm{O} 10$ & $0.8859(9)$ & $0.4468(10)$ & $0.6196(9)$ & 1 & $0.029(3)$ \\
\hline $\mathrm{O} 11$ & $0.9030(8)$ & $0.4366(8)$ & $0.3952(7)$ & 1 & $0.019(2)$ \\
\hline $\mathrm{O} 12$ & $0.7289(9)$ & $0.3276(8)$ & $0.4385(9)$ & 1 & $0.019(2)$ \\
\hline $\mathrm{O} 13$ & $0.1983(9)$ & $0.4965(9)$ & $-0.0648(8)$ & 1 & $0.029(2)$ \\
\hline $\mathrm{O} 14$ & $0.3579(9)$ & $0.6411(7)$ & $0.0257(9)$ & 1 & $0.017(2)$ \\
\hline $\mathrm{O} 15$ & $0.3653(8)$ & $0.5281(8)$ & $-0.1668(8)$ & 1 & $0.020(2)$ \\
\hline O16 & $0.5531(8)$ & $0.5057(8)$ & $0.0339(7)$ & 1 & $0.019(2)$ \\
\hline $\mathrm{O} 17$ & $0.3681(9)$ & $0.3582(7)$ & $-0.0433(10)$ & 1 & $0.019(2)$ \\
\hline $\mathrm{O} 18$ & $-0.1507(10)$ & $0.6112(8)$ & $-0.0222(10)$ & 1 & $0.022(2)$ \\
\hline $\mathrm{O} 20$ & $-0.0714(9)$ & $0.4579(8)$ & $-0.1056(9)$ & 1 & $0.024(2)$ \\
\hline O19 & $-0.3062(8)$ & $0.4538(8)$ & $-0.0954(8)$ & 1 & $0.017(2)$ \\
\hline $\mathrm{O} 21$ & $0.0176(8)$ & $0.4864(9)$ & $0.1510(7)$ & 1 & $0.019(2)$ \\
\hline $\mathrm{O} 22$ & $0.8050(8)$ & $0.4919(8)$ & $0.1544(7)$ & 1 & $0.0147(17)$ \\
\hline $\mathrm{O} 23$ & $-0.1323(9)$ & $0.3279(8)$ & $0.0399(9)$ & 1 & $0.020(2)$ \\
\hline $\mathrm{O} 24$ & $1.1431(10)$ & $0.4419(10)$ & $0.3995(9)$ & 1 & $0.032(3)$ \\
\hline $\mathrm{O} 25$ & $1.0019(11)$ & $0.6020(9)$ & $0.3365(10)$ & 1 & $0.028(3)$ \\
\hline $\mathrm{O} 26$ & $0.9672(10)$ & $0.3151(8)$ & $0.2474(9)$ & 1 & $0.023(2)$ \\
\hline $\mathrm{O} 27$ & $0.5779(11)$ & $0.6308(8)$ & $-0.1493(9)$ & 1 & $0.024(2)$ \\
\hline $\mathrm{C} 1$ & $0.4831(10)$ & $0.4880(9)$ & $-0.3193(8)$ & 1 & $0.026(2)$ \\
\hline $\mathrm{C} 2$ & $0.4856(10)$ & $0.3513(8)$ & $-0.1529(10)$ & 1 & $0.023(2)$ \\
\hline $\mathrm{C} 3$ & $0.3206(12)$ & $0.8446(10)$ & $0.3249(11)$ & 1 & $0.033(3)$ \\
\hline $\mathrm{C} 4$ & $0.1646(13)$ & $0.6721(11)$ & $0.1956(13)$ & 1 & $0.045(4)$ \\
\hline N1 & $0.3664(17)$ & $0.3008(11)$ & $0.5647(13)$ & 1 & $0.057(4)$ \\
\hline N2 & $0.1964(11)$ & $0.3487(10)$ & $0.1401(12)$ & 1 & $0.036(3)$ \\
\hline N3 & $0.2771(11)$ & $0.1606(10)$ & $0.3192(12)$ & 1 & $0.033(3)$ \\
\hline OB1 & $0.1950(18)$ & $0.6527(19)$ & $-0.3271(17)$ & 1 & $0.098(11)$ \\
\hline $\mathrm{C} 5$ & $0.019(2)$ & $0.2745(16)$ & $0.5357(19)$ & 1 & $0.090(7)$ \\
\hline C6 & $0.1775(19)$ & $0.4146(17)$ & $-0.3431(19)$ & 1 & $0.097(8)$ \\
\hline $\mathrm{C} 7$ & $0.0472(15)$ & $0.3021(15)$ & $-0.153(2)$ & 1 & $0.086(7)$ \\
\hline $\mathrm{C} 8$ & $0.6186(12)$ & $0.2999(10)$ & $0.4139(11)$ & 1 & $0.013(2)$ \\
\hline N4 & $0.5945(15)$ & $0.2001(11)$ & $0.4473(14)$ & 1 & $0.023(3)$ \\
\hline N5 & $0.4088(14)$ & $0.3170(10)$ & $-0.1140(13)$ & 1 & $0.020(3)$ \\
\hline N6 & $0.3559(16)$ & $0.2210(10)$ & $-0.1635(14)$ & 1 & $0.021(3)$ \\
\hline OB2 & $-0.0733(12)$ & $0.2826(10)$ & $0.1364(13)$ & 1 & $0.016(3)$ \\
\hline C9 & $-0.0497(15)$ & $0.1790(11)$ & $0.1189(12)$ & 1 & $0.021(3)$ \\
\hline $\mathrm{C} 10$ & $0.7112(13)$ & $0.1491(12)$ & $0.5247(13)$ & 1 & $0.032(3)$ \\
\hline $\mathrm{C} 11$ & $0.2678(12)$ & $0.1889(10)$ & $-0.1105(12)$ & 1 & $0.023(3)$ \\
\hline $\mathrm{C} 12$ & $0.0049(12)$ & $0.1229(10)$ & $0.2371(11)$ & 1 & $0.026(3)$ \\
\hline N7 & $1.1388(4)$ & $0.2607(2)$ & $0.3876(2)$ & 1 & $0.0374(8)$ \\
\hline N8 & $1.2418(3)$ & $0.3516(2)$ & $0.3046(2)$ & 1 & $0.0324(8)$ \\
\hline N9 & $1.0559(3)$ & $0.2138(2)$ & $0.22544(19)$ & 1 & $0.0267(7)$ \\
\hline OB3 & $1.0700(3)$ & $0.4291(2)$ & $0.37050(17)$ & 1 & $0.0249(7)$ \\
\hline $\mathrm{C} 13$ & $0.8926(3)$ & $0.2830(2)$ & $0.2951(2)$ & 1 & $0.0293(7)$ \\
\hline
\end{tabular}


Table 2. (continued)

\begin{tabular}{|c|c|c|c|c|c|}
\hline Atoms & $x$ & $y$ & $z$ & Occupation & $U_{\text {éq }}$ \\
\hline $\mathrm{C} 14$ & $1.0084(3)$ & $0.39953(18)$ & $0.22311(17)$ & 1 & $0.0191(6)$ \\
\hline C15 & $0.6742(3)$ & $0.2106(2)$ & $-0.0488(2)$ & 1 & $0.0318(8)$ \\
\hline $\mathrm{C} 16$ & $0.6148(3)$ & $0.0641(2)$ & $-0.0005(2)$ & 1 & $0.0282(7)$ \\
\hline N10 & $0.6321(3)$ & $0.22837(19)$ & $0.09622(19)$ & 1 & $0.0227(6)$ \\
\hline N11 & $0.8690(3)$ & $0.11165(19)$ & $0.01905(19)$ & 1 & $0.0231(7)$ \\
\hline N12 & $0.8830(3)$ & $0.26868(18)$ & $0.09379(18)$ & 1 & $0.0194(6)$ \\
\hline OB4 & $0.8215(3)$ & $0.13008(19)$ & $0.14410(18)$ & 1 & $0.0218(6)$ \\
\hline $\mathrm{C} 17$ & $1.0048(4)$ & $0.6015(2)$ & $0.3963(2)$ & 1 & $0.0334(8)$ \\
\hline C18 & $1.1599(3)$ & $0.5630(2)$ & $0.3066(2)$ & 1 & $0.0291(7)$ \\
\hline C19 & $0.9046(3)$ & $0.55980(19)$ & $0.23112(17)$ & 1 & $0.0197(6)$ \\
\hline $\mathrm{C} 20$ & $0.8216(3)$ & $0.45809(19)$ & $0.30340(17)$ & 1 & $0.0209(6)$ \\
\hline N13 & $1.0531(4)$ & $0.0517(2)$ & $0.1276(3)$ & 1 & $0.0421(10)$ \\
\hline N14 & $1.1178(3)$ & $0.2044(2)$ & $0.0885(2)$ & 1 & $0.0341(8)$ \\
\hline N15 & $0.6985(4)$ & $0.6144(2)$ & $0.2837(2)$ & 1 & $0.0339(8)$ \\
\hline OB5 & $0.6798(4)$ & $0.5809(2)$ & $0.1290(2)$ & 1 & $0.0347(8)$ \\
\hline $\mathrm{C} 21$ & $0.5735(3)$ & $0.4532(2)$ & $0.19150(19)$ & 1 & $0.0238(7)$ \\
\hline $\mathrm{C} 22$ & $0.7542(3)$ & $0.40430(19)$ & $0.14382(18)$ & 1 & $0.0197(6)$ \\
\hline N16 & $0.4175(3)$ & $0.2881(2)$ & $0.1314(2)$ & 1 & $0.0288(7)$ \\
\hline N17 & $0.5100(3)$ & $0.3611(2)$ & $0.0356(2)$ & 1 & $0.0302(7)$ \\
\hline N18 & $0.6423(3)$ & $0.3062(2)$ & $0.24609(18)$ & 1 & $0.0244(7)$ \\
\hline $\mathrm{C} 23$ & $0.9406(3)$ & $0.4371(2)$ & $0.07517(18)$ & 1 & $0.0241(7)$ \\
\hline $\mathrm{C} 24$ & $0.7780(4)$ & $0.3593(3)$ & $0.4019(2)$ & 1 & $0.0398(9)$ \\
\hline OB6 & $1.2867(5)$ & $0.6372(3)$ & $0.1724(3)$ & 1 & $0.0324(11)$ \\
\hline OW1 & $1.2973(5)$ & $0.4774(4)$ & $0.0692(3)$ & 1 & $0.0324(11)$ \\
\hline OW2 & $1.2590(4)$ & $0.4744(3)$ & $0.1361(3)$ & 1 & $0.0278(10)$ \\
\hline OW3 & $1.3283(5)$ & $0.5586(4)$ & $0.0573(3)$ & 1 & $0.0377(12)$ \\
\hline OW4 & $1.3233(5)$ & $0.6361(3)$ & $0.1070(3)$ & 0.59 & $0.0381(10)$ \\
\hline OW5 & $1.2565(4)$ & $0.5542(3)$ & $0.1845(2)$ & 0.41 & $0.0299(9)$ \\
\hline OW6 & $1.2264(4)$ & $0.4006(3)$ & $0.1541(3)$ & 0.42 & $0.0342(10)$ \\
\hline OW7 & $1.2771(4)$ & $0.7060(3)$ & $0.2166(2)$ & 0.58 & $0.0468(10)$ \\
\hline OW8 & $0.7018(5)$ & $-0.0659(3)$ & $0.1858(3)$ & 0.62 & $0.0352(12)$ \\
\hline OW9 & $0.7521(4)$ & $-0.1023(3)$ & $0.0646(3)$ & 0.38 & $0.0255(9)$ \\
\hline OW10 & $0.7076(5)$ & $-0.1936(3)$ & $0.0542(3)$ & 1 & $0.0322(11)$ \\
\hline
\end{tabular}

with the molybdate ring. In turn, two of these oxo-groups adopt the $\mu_{2}$-bridging mode, linking one molybdenum site and the arsenic, and the other one adopts the $\mu_{3}$-bridging mode, linking two molybdenum sites and the arsenic, while the As1 subunit shares three oxo-groups of six octahedral $\mathrm{MoO}_{6}$.

In these anions there are three categories of Mo-O bonds. Thus, the Mo is bonded to two terminal oxygen atoms with Mo-O distances in the $1.698(3)-1.736(3) \AA$ range. The Mo-O bonds ranging from 1.891(3) to 2.256(3) $\AA$ are found with oxygen atoms bonded to two molybdenum atoms. The longest Mo-O distances, 2.198(3)-2.349(3) ^, appear with oxygen atoms coordinated to arsenic and molybdenum. The six-member ring is nearly planar with a mean deviation of $0.1775 \AA$, and the interatomic distance between As1 and As2 is 3.89(1) $\AA$ with the orientation of the As1-As2 vector to the ring being $57.9^{\circ}$. As atoms are surrounded by four oxygen atoms and exhibit distorted geometry, with As-O bond ranging from $1.646(3)$ to $1.715(3) \AA$ and from $1.668(3)$ to
1.719(3) $\AA$ for As1 and As2, respectively. The O-As-O bond angles between $105.16(3)^{\circ}$ and $114.53(3)^{\circ}$.

The title polyanion differs from a well-known Lindqvist type of structure $\left[\mathrm{Mo}_{6} \mathrm{O}_{19}\right]^{2-},{ }^{24}$ in which each Mo atom of the polyanion is coordinated by six $\mathrm{O}$ atoms (one terminal $\mathrm{O}_{t}$, one central $\mathrm{O}_{c}$ and four bridging $\mathrm{O}_{b}$ ) in a distorted octahedral arrangement, due to the displacement of Mo atoms towards the terminal $\mathrm{O}_{\mathrm{t}}$ atoms. The six $\mathrm{MoO}_{6}$ octahedra share a common vertex at the central $\mathrm{O}_{\mathrm{c}}$, and also each octahedron shares four edges with adjacent octahedral ${ }^{25}$ and thus exhibits an approximate $\mathrm{O}_{\mathrm{h}}$ symmetry, whereas the $\left[\left(\mathrm{HAsO}_{4}\right)_{2} \mathrm{Mo}_{6} \mathrm{O}_{19}\right]^{6-}$ cluster has approximate $\mathrm{C}_{1}$ symmetry.

BVS calculations ${ }^{26}$ (http://kristall. uni.mki.gwdg.de/softbv) revealed that all the molybdenum atoms have valence sums ranging from 5.942 to 6.055 , with an average value of 6.001 , close to the ideal value of 6 for $\mathrm{Mo}^{\mathrm{VI}}$. The calculated average value of arsenic (V), oxygen (-II) atoms are 4.940 and 1.973, respectively. Moreover, these calculations indicated that the bridging oxygen atoms, $\mathrm{O} 26$ and $\mathrm{O} 27$, are protonated. 
Table 3. Selected bond lengths $(\AA)$.

\begin{tabular}{|c|c|}
\hline \multicolumn{2}{|l|}{ Bond lengths } \\
\hline Mo1-O1 & 1.701(3) \\
\hline Mo1-O2 & $1.729(3)$ \\
\hline Mo1-O3 & $1.890(3)$ \\
\hline Mo1-O4 & $1.976(3)$ \\
\hline Mo1-O5 & $2.195(3)$ \\
\hline Mo1-O6 & $2.345(3)$ \\
\hline Mo2-O7 & $1.712(3)$ \\
\hline Mo2-O8 & $1.720(3)$ \\
\hline Mo2-O9 & 1.899(3) \\
\hline Mo2-O10 & $1.946(3)$ \\
\hline Mo2-O11 & $2.259(3)$ \\
\hline Mo2-O12 & $2.254(3)$ \\
\hline Mo3-O13 & $1.716(3)$ \\
\hline Mo3-O14 & $1.734(3)$ \\
\hline Mo3-O4 & $1.902(3)$ \\
\hline Mo3-O15 & $1.947(3)$ \\
\hline Mo3-O16 & $2.264(3)$ \\
\hline Mo3-O6 & $2.285(3)$ \\
\hline Mo4-O17 & 1.701(3) \\
\hline Mo4-O18 & $1.725(3)$ \\
\hline Mo4-O3 & $1.890(3)$ \\
\hline Mo4-O10 & $1.961(3)$ \\
\hline Mo4-O12 & $2.225(3)$ \\
\hline Mo4-O11 & $2.316(3)$ \\
\hline Mo5-O19 & $1.698(3)$ \\
\hline Mo5-O20 & $1.738(3)$ \\
\hline Mo5-O21 & $1.918(3)$ \\
\hline Mo5-O15 & $1.942(3)$ \\
\hline Mo5-O22 & $2.222(3)$ \\
\hline Mo5-O16 & $2.354(3)$ \\
\hline Mo6-O24 & $1.702(3)$ \\
\hline Mo6-O23 & $1.713(3)$ \\
\hline Mo6-O9 & $1.915(3)$ \\
\hline Mo6-O21 & $1.949(3)$ \\
\hline Mo6-O22 & $2.271(3)$ \\
\hline Mo6-O25 & $2.300(3)$ \\
\hline As1-O26 & $1.646(3)$ \\
\hline As1-O11 & $1.691(3)$ \\
\hline As1-O6 & $1.711(3)$ \\
\hline As1-O22 & $1.715(3)$ \\
\hline As2-O5 & $1.668(3)$ \\
\hline As2-O25 & $1.668(3)$ \\
\hline As2-O16 & $1.696(3)$ \\
\hline As2-O27 & $1.719(3)$ \\
\hline $\mathrm{C} 1-\mathrm{OB} 1$ & $1.226(6)$ \\
\hline $\mathrm{C} 1-\mathrm{N} 1$ & $1.372(7)$ \\
\hline $\mathrm{C} 1-\mathrm{N} 2$ & $1.378(6)$ \\
\hline $\mathrm{C} 2-\mathrm{C} 4$ & $1.353(7)$ \\
\hline C2-C3 & $1.419(7)$ \\
\hline $\mathrm{C} 3-\mathrm{N} 3$ & $1.306(6)$ \\
\hline C3-N2 & $1.368(6)$ \\
\hline C4-N1 & $1.360(7)$ \\
\hline $\mathrm{C} 5-\mathrm{OB} 2$ & $1.218(7)$ \\
\hline C5-N5 & $1.368(6)$ \\
\hline $\mathrm{C} 5-\mathrm{N} 4$ & $1.378(6)$ \\
\hline C6-N6 & $1.322(6)$ \\
\hline C6-N6 & $1.353(6)$ \\
\hline C6-C7 & $1.418(6)$ \\
\hline
\end{tabular}

Table 3. (continued)

\begin{tabular}{|c|c|}
\hline \multicolumn{2}{|l|}{ Bond lengths } \\
\hline C7-C8 & $1.352(8)$ \\
\hline C8-N5 & $1.357(7)$ \\
\hline C9-OB3 & $1.223(6)$ \\
\hline C9-N7 & $1.367(7)$ \\
\hline C9-N8 & $1.384(7)$ \\
\hline C10-N9 & $1.309(6)$ \\
\hline C10-N8 & $1.357(6)$ \\
\hline C10-C11 & $1.438(7)$ \\
\hline $\mathrm{C} 11-\mathrm{C} 12$ & $1.328(8)$ \\
\hline C12-N7 & $1.360(7)$ \\
\hline C13-OB4 & $1.213(7)$ \\
\hline C13-N10 & $1.372(6)$ \\
\hline C13-N11 & $1.376(7)$ \\
\hline C14-N12 & $1.307(6)$ \\
\hline C14-N10 & $1.367(6)$ \\
\hline C14-C15 & $1.430(7)$ \\
\hline C15-C16 & $1.352(8)$ \\
\hline C16-N11 & $1.351(8)$ \\
\hline C17-OB5 & $1.246(8)$ \\
\hline C17-N13 & $1.348(8)$ \\
\hline C17-N14 & $1.365(8)$ \\
\hline C18-N15 & $1.321(8)$ \\
\hline C18-N13 & $1.356(8)$ \\
\hline C18-C19 & $1.432(8)$ \\
\hline C19-C20 & $1.325(10)$ \\
\hline C20-N14 & $1.364(9)$ \\
\hline C21-N17 & $1.317(6)$ \\
\hline C21-N16 & $1.366(6)$ \\
\hline $\mathrm{C} 21-\mathrm{C} 23$ & $1.424(6)$ \\
\hline C22-OB6 & $1.215(6)$ \\
\hline C22-N18 & $1.366(6)$ \\
\hline C22-N16 & $1.382(6)$ \\
\hline N18-C24 & $1.378(7)$ \\
\hline $\mathrm{C} 23-\mathrm{C} 24$ & $1.337(7)$ \\
\hline
\end{tabular}

In the structure the $\mathrm{Cyt}^{+}$ions, acting as chargecompensating cations and space-filling structural subunits, are connected to the three-dimensional (3D) supramolecular by $\mathrm{N}-\mathrm{H} \cdots \mathrm{O}$ and $\mathrm{O}-\mathrm{H} \cdots \mathrm{N}$ hydrogen bonds and aromatic $\pi-\pi$ stacking interactions.

The arrangement of the $\left[\left(\mathrm{HAsO}_{4}\right)_{2} \mathrm{Mo}_{6} \mathrm{O}_{19}\right]^{6-}$ clusters and the cytosinium cations along the $b$ axis is demonstrated in figure 4. As listed in table 4, OW-H... O, OW-H...OW and $\mathrm{OW}-\mathrm{H} \cdots \mathrm{N}$ hydrogen bonds between the solvent water molecules, $\mathrm{Cyt}^{+}$cations and the clusters have interatomic $\mathrm{O} \cdots \mathrm{O}$ and $\mathrm{N} \cdots \mathrm{O}$ distances ranging, respectively, from 2.648 to $3.405 \AA$ and from 2.672 to $3.283 \AA$ and the hydrogen-bond angles from $110.37^{\circ}$ to $179.45^{\circ}$. It is noticeable that there also exist the $\mathrm{N}-\mathrm{H} \cdots \mathrm{N}$ hydrogen bonds between two organic amines with interatomic distances between 2.672 and $3.283 \AA$ and the close contact distance between some adjacent aromatic rings of $\mathrm{Cyt}^{+}$contributes to the formation of the extensive $\pi-\pi$ interactions (figure 5 ). These hydrogen bonds hold the components together into a 3D network and make the crystal structure of the compound more stable. 


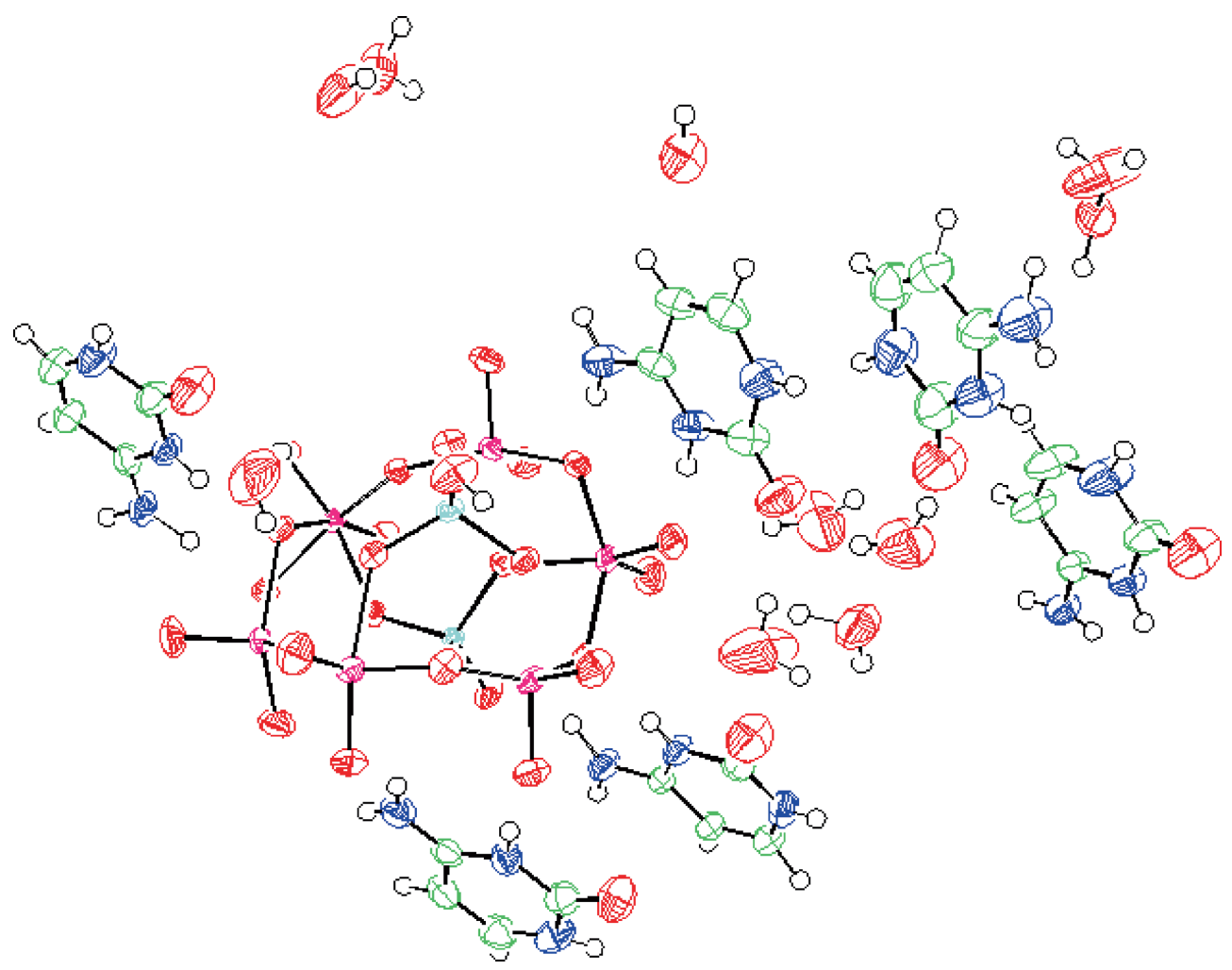

Figure 2. An ORTEP drawing of the asymmetric unit.
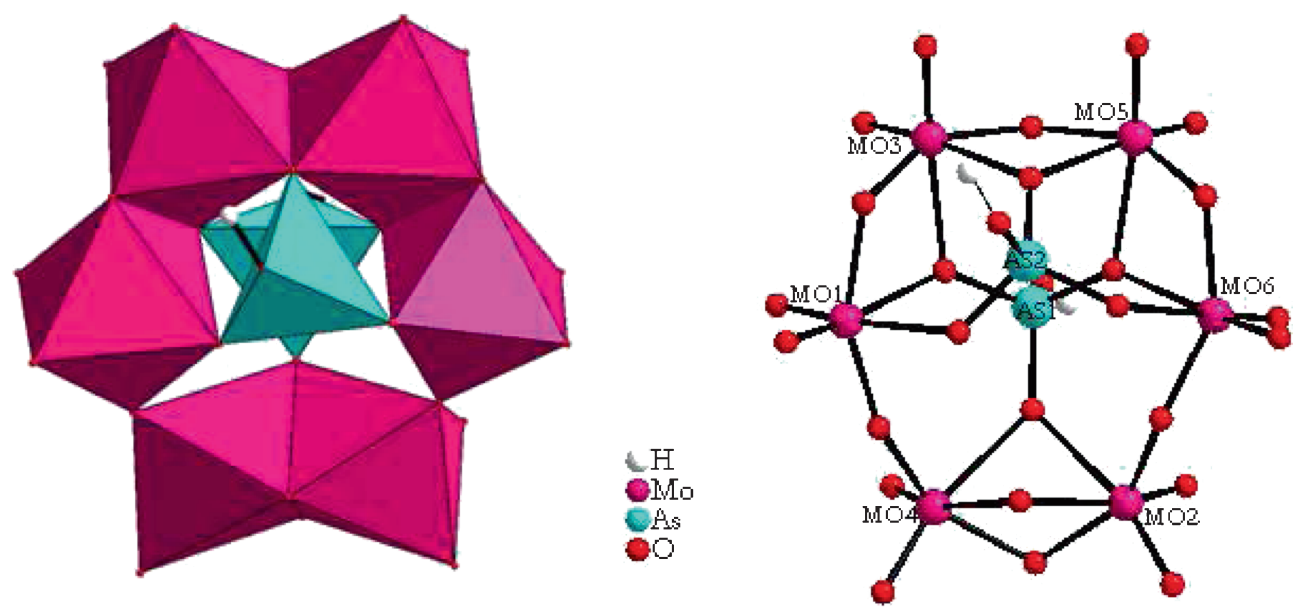

Figure 3. A polyhedral representation of the $\left[\left(\mathrm{HAsO}_{4}\right)_{2} \mathrm{Mo}_{6} \mathrm{O}_{19}\right]^{6-}$ anion.

\subsection{IR absorption spectrometry}

Vibrational spectrum exhibits the characteristic bands associated with the building constituents from which the title material was synthesized: the polyoxoanion $\left[\left(\mathrm{HAsO}_{4}\right)_{2} \mathrm{Mo}_{6} \mathrm{O}_{19}\right]^{6-}$, organic groups and water molecules. The IR spectrum of the title compound (figure 6) has some characteristic bands for the cytosine moiety. ${ }^{27}$ The broad absorption observed in the region between 3200 and 2800 $\mathrm{cm}^{-1}$ can be assigned to the $\mathrm{O}-\mathrm{H}, \mathrm{N}-\mathrm{H}$ and $\mathrm{C}-\mathrm{H}$ stretching modes. Absorptions in $1620-1674 \mathrm{~cm}^{-1}$ are attributed to the stretching vibration of $\mathrm{C}=\mathrm{O}$ and bending vibration of the $\mathrm{OH}$ group of the water molecules. On the other hand, the title compound exhibits bands in the $1532-1100 \mathrm{~cm}^{-1}$ region, corresponding to the stretching vibrations $\mathrm{C}-\mathrm{C}$ and $\mathrm{C}-\mathrm{N}$ and bending vibration $\mathrm{C}-\mathrm{C}-\mathrm{H}$. The low-wavenumber characteristic peaks at $923,876,828,781,668,621,574$, 515 and $480 \mathrm{~cm}^{-1}$ are attributed to the $\left[\left(\mathrm{HAsO}_{4}\right)_{2} \mathrm{Mo}_{6} \mathrm{O}_{19}\right]^{6-}$ polyoxoanions. ${ }^{28}$

\subsection{UV-visible spectra}

The UV-visible absorption behaviour of the title compound was analysed in the 190-800 $\mathrm{nm}$ range using an aqueous 


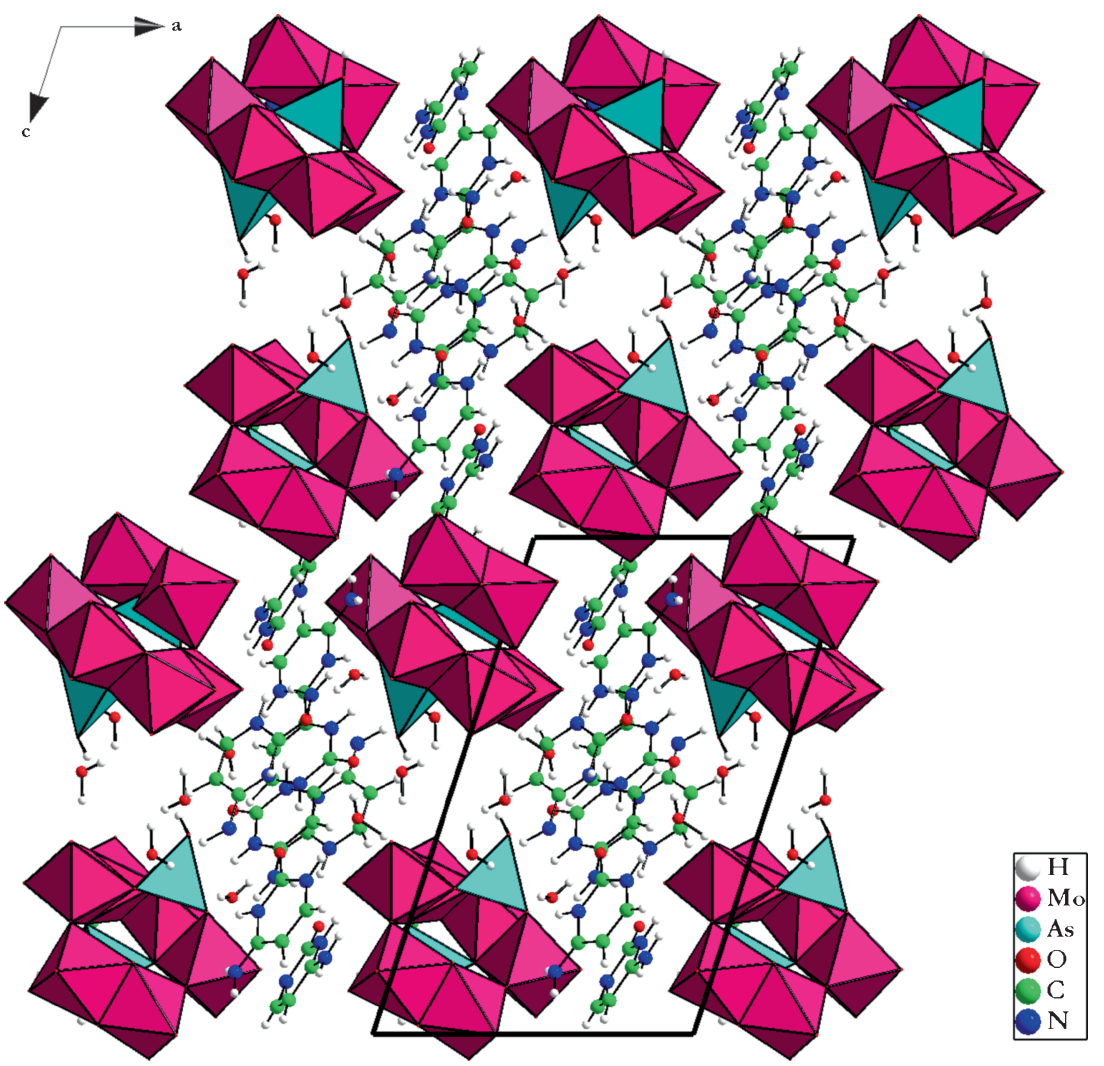

Figure 4. A packing view of compound along the [010] direction, showing the arrangement of the $\left[\left(\mathrm{HAsO}_{4}\right) \mathrm{Mo}_{6} \mathrm{O}_{19}\right]^{6-}$ clusters, cytosinium cations and the lattice water molecules in the crystal structure.

Table 4. Hydrogen-bonding geometry $\left(\AA{ }^{\circ}\right)$.

\begin{tabular}{|c|c|c|c|c|}
\hline $\mathrm{D}-\mathrm{H} \cdots \mathrm{A}$ & D-H $(\AA)$ & $\mathrm{H} \cdots \mathrm{A}(\AA)$ & $\mathrm{D} \cdots \mathrm{A}(\AA)$ & $\mathrm{D}-\mathrm{H} \cdots \mathrm{A}\left({ }^{\circ}\right)$ \\
\hline O26-HAS1 $\cdots$ N17 $7^{\mathrm{i}}$ & 0.821 & 2.171 & 2.846 & 139.52 \\
\hline O $27-H A S 2 \cdots$ OW $1^{\text {ii }}$ & 0.959 & 1.830 & 2.648 & 141.46 \\
\hline $\mathrm{N} 3-\mathrm{H} 1 \cdots \mathrm{O} 23^{\mathrm{iii}}$ & 0.756 & 2.377 & 2.864 & 123.37 \\
\hline 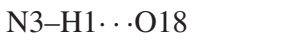 & 0.756 & 2.494 & 3.048 & 131.42 \\
\hline N3-H4 . . O6 & 0.914 & 2.355 & 3.029 & 130.49 \\
\hline $\mathrm{N} 5-\mathrm{H} 11 \cdots \mathrm{OW} 2^{\text {iv }}$ & 1.014 & 1.933 & 2.681 & 128.24 \\
\hline N6-H12..O17 & 1.146 & 2.575 & 3.240 & 115.57 \\
\hline N8-H14. . O20 & 0.943 & 1.803 & 2.745 & 177.63 \\
\hline $\mathrm{N} 7-\mathrm{H} 15 \cdots \mathrm{OB} 4^{\mathrm{v}}$ & 0.956 & 1.783 & 2.672 & 153.40 \\
\hline $\mathrm{N} 11-\mathrm{H} 21 \cdots \mathrm{OW} 6^{\mathrm{vii}}$ & 0.932 & 2.452 & 3.000 & 117.62 \\
\hline 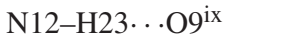 & 0.740 & 2.152 & 2.814 & 149.35 \\
\hline $\mathrm{N} 12-\mathrm{H} 23 \cdots \mathrm{O} 23^{\mathrm{ix}}$ & 0.740 & 2.646 & 3.224 & 136.75 \\
\hline $\mathrm{N} 13-\mathrm{H} 26 \cdots \mathrm{N} 13^{\mathrm{v}}$ & 0.862 & 2.257 & 2.889 & 130.14 \\
\hline $\mathrm{N} 13-\mathrm{H} 26 \cdots \mathrm{OW} 4^{\mathrm{v}}$ & 0.862 & 2.594 & 3.273 & 136.43 \\
\hline $\mathrm{N} 15-\mathrm{H} 28 \cdots \mathrm{OW}^{\mathrm{ii}}$ & 1.025 & 2.056 & 3.022 & 156.19 \\
\hline $\mathrm{N} 15-\mathrm{H} 28 \cdots \mathrm{OB} 2^{\mathrm{ii}}$ & 1.025 & 2.577 & 3.283 & 125.70 \\
\hline $\mathrm{N} 17-\mathrm{H} 35 \cdots \mathrm{O} 26$ & 1.132 & 1.741 & 2.699 & 138.90 \\
\hline OW3-H1W3...O5 & 0.868 & 2.486 & 2.905 & 110.37 \\
\hline OW4-H1W4. .N11 ${ }^{\mathrm{v}}$ & 0.798 & 2.192 & 2.844 & 139.07 \\
\hline OW5-H1W5 . O $1^{\mathrm{x}}$ & 1.074 & 2.244 & 2.996 & 125.37 \\
\hline OW5-H2W5 . .OW8 & 0.855 & 2.109 & 2.693 & 125.07 \\
\hline OW7-H1W7. .O17 vi & 0.994 & 2.319 & 3.061 & 130.66 \\
\hline OW9-H1W9... OW $7^{\text {ix }}$ & 0.855 & 2.119 & 2.849 & 142.96 \\
\hline OW9-H2W9. . OW4 ${ }^{\mathrm{v}}$ & 0.990 & 2.263 & 3.069 & 137.81 \\
\hline
\end{tabular}

${ }^{\mathrm{i}}-x+2, y+1,-z ;{ }^{\mathrm{ii}}-x+1, y+1,-z+1 ;{ }^{\mathrm{iii}} x+1, y, z ;{ }^{\mathrm{iv}} x, y-1, z ;{ }^{\mathrm{v}}-x+1,-y+2$, $-z+1 ;{ }^{\mathrm{vi}} x-1, y, z ;{ }^{\mathrm{vii}} x, y+1, z ;{ }^{\mathrm{ix}} x, y+1, z ;{ }^{\mathrm{x}}-x+2,-y+1,-z+1$. 


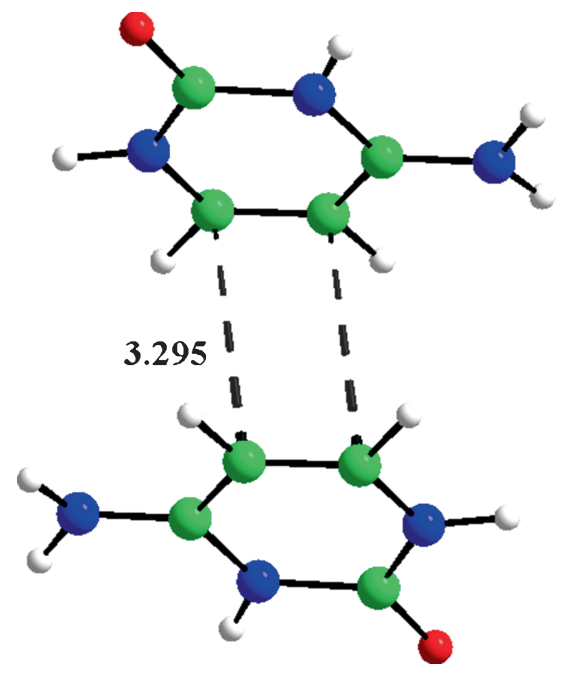

Figure 5. The $\pi-\pi$ interactions in the compound.

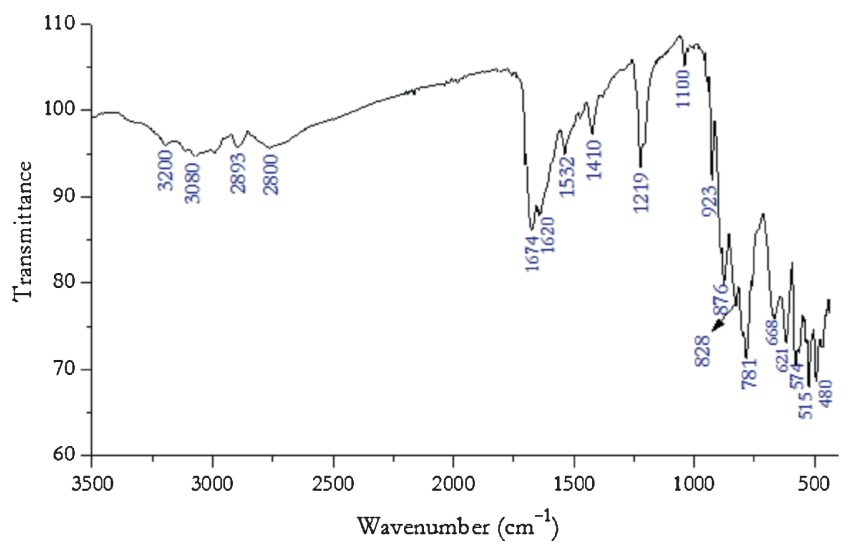

Figure 6. FT-IR spectrum of $\left(\mathrm{C}_{4} \mathrm{H}_{6} \mathrm{~N}_{3} \mathrm{O}\right)_{6}\left[\left(\mathrm{HAsO}_{4}\right)_{2} \mathrm{Mo}_{6} \mathrm{O}_{19}\right]$. $7 \mathrm{H}_{2} \mathrm{O}$ between 4000 and $400 \mathrm{~cm}^{-1}$.

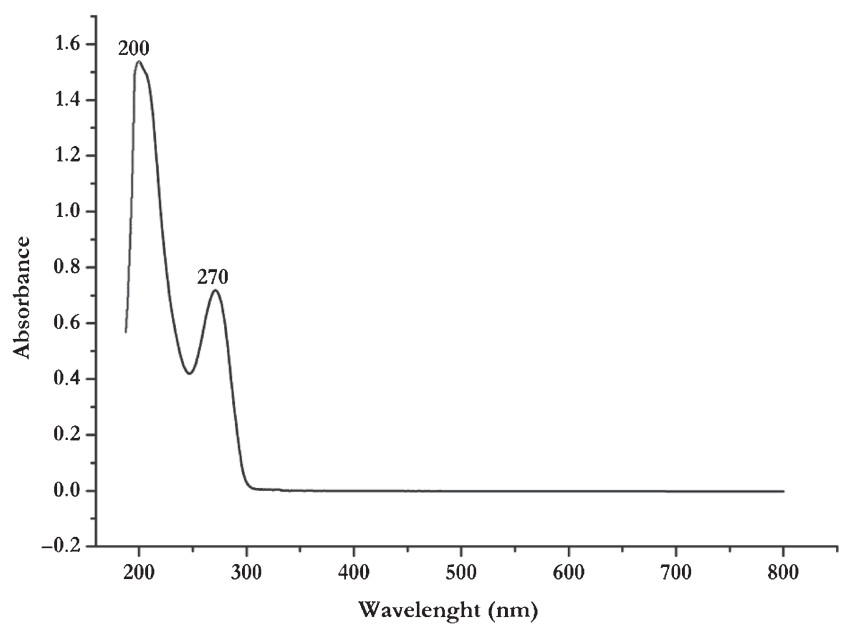

Figure 7. UV-visible absorption spectrum of $\left(\mathrm{C}_{4} \mathrm{H}_{6} \mathrm{~N}_{3} \mathrm{O}\right)_{6}$ $\left[\left(\mathrm{HAsO}_{4}\right)_{2} \mathrm{Mo}_{6} \mathrm{O}_{19}\right] \cdot 7 \mathrm{H}_{2} \mathrm{O}$.

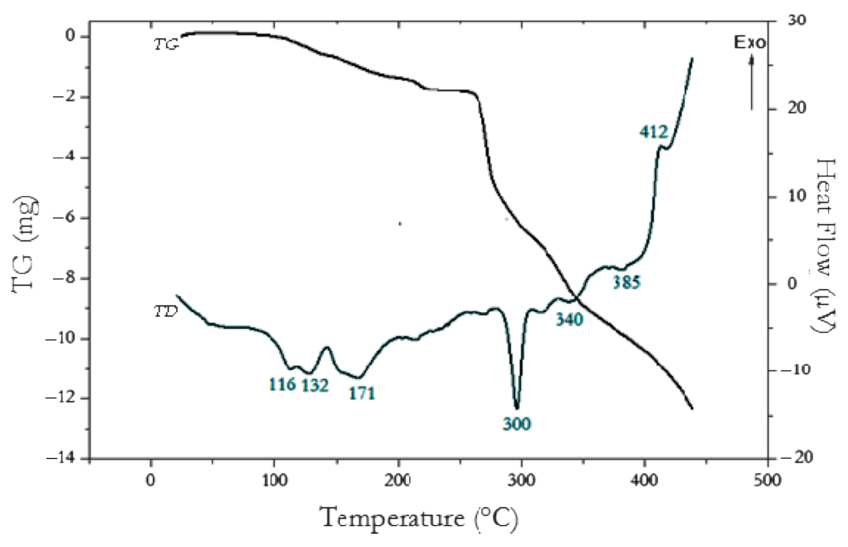

Figure 8. The two curves corresponding to the DTA and TGA analysis of $\left(\mathrm{C}_{4} \mathrm{H}_{6} \mathrm{~N}_{3} \mathrm{O}\right)_{6}\left[\left(\mathrm{HAsO}_{4}\right)_{2} \mathrm{Mo}_{6} \mathrm{O}_{19}\right] \cdot 7 \mathrm{H}_{2} \mathrm{O}$.

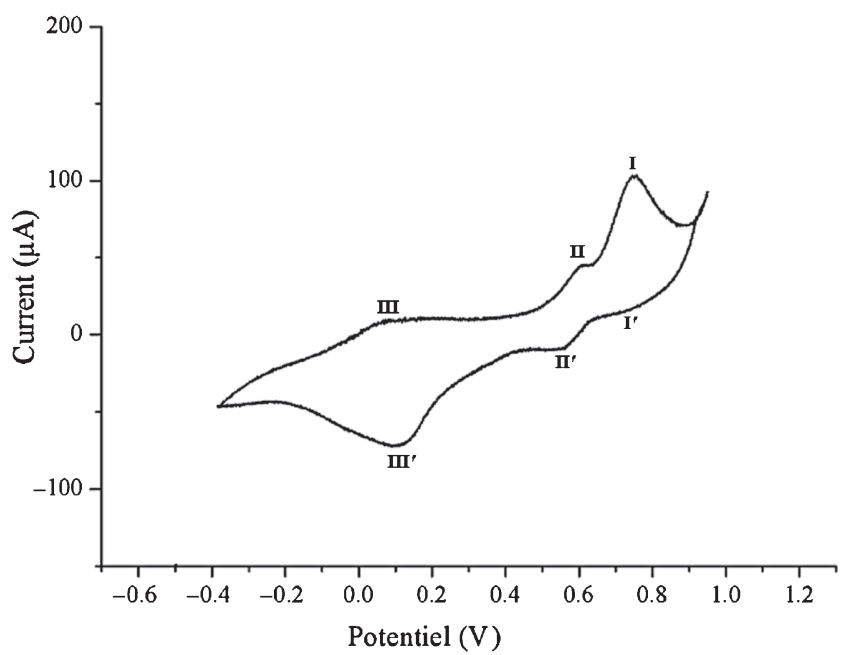

Figure 9. Cyclic voltammogram of compound in $1 \mathrm{~mol}^{-1}$ $\mathrm{H}_{2} \mathrm{SO}_{4}$ solution at a scan rate of $100 \mathrm{mV} \mathrm{s}^{-1}$.

solution. The electronic spectrum of compound is shown in figure 7, giving two evident bands at 200 and $270 \mathrm{~nm}$. They are attributed to the ligand-to-metal charge transfers of Ot-Mo and $\mu-\mathrm{O}-\mathrm{Mo}$, respectively, where electrons are promoted from the low-energy electronic states, mainly comprised of oxygen $2 p$ orbitals, to the high-energy states, which are mainly comprised of metal $d$ orbitals. ${ }^{29-31}$

\subsection{Thermal analysis}

The two curves corresponding to the DTA and TGA analysis of $\left(\mathrm{C}_{4} \mathrm{H}_{6} \mathrm{~N}_{3} \mathrm{O}\right)_{6}\left[\left(\mathrm{HAsO}_{4}\right)_{2} \mathrm{Mo}_{6} \mathrm{O}_{19}\right] \cdot 7 \mathrm{H}_{2} \mathrm{O}$ are given in figure 8 . The DTA curve shows that this compound undertakes a series of endothermic and exothermic peaks in a wide temperature range $\left(100-420^{\circ} \mathrm{C}\right)$. The DTA curve shows three endothermic peaks characterized by a weight loss of $8.5 \%$ observed on the TGA curve in the range $100-200^{\circ} \mathrm{C}$. This thermal phenomenon corresponds to the loss of seven water 
molecules. The DTA curve exhibits also several endothermic peaks between 300 and $400^{\circ} \mathrm{C}$, which can be attributed to the degradation of the organic moiety.

\subsection{Electrochemical property}

The redox behaviour of the title compound was investigated by cyclic voltammogram in the $1 \mathrm{M} \mathrm{H}_{2} \mathrm{SO}_{4}$ aqueous solution, shown in figure 9 . It can be seen that in the potential range from -600 to $1100 \mathrm{mV}$, three quasi-reversible redox peaks appear and the mean peak potentials $E_{1 / 2}=\left(E_{\mathrm{pa}}+E_{\mathrm{pc}}\right) / 2$ are $0.741(\mathrm{I}), 0.570$ (II) and $0.086(\mathrm{III}) \mathrm{mV}$. The I-I', II-II' and III-III' should be ascribed to three consecutive two-electron processes of molybdenum. ${ }^{32,33}$

\section{Conclusion}

It is important to remark that polyanions consisting of a ring of six polyhedral $\operatorname{MoO}_{x}(x=5,6)$ capped above and below by arsenate groups are limited to the three isomers of $\left[\mathrm{H}_{x} \mathrm{As}_{2} \mathrm{Mo}_{6} \mathrm{O}_{26}\right]^{(6-x)-},\left[\mathrm{As}^{\mathrm{V}}{ }_{2} \mathrm{Mo}_{6} \mathrm{O}_{26}\right]^{6-}$,

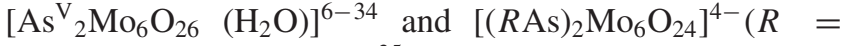
$\left.\mathrm{CH}_{3}, \mathrm{C}_{6} \mathrm{H}_{5}, \mathrm{p}-\mathrm{C}_{6} \mathrm{H}_{4} \mathrm{NH}_{2}\right) .{ }^{35}$ In summary new compounds, including $\left[\left(\mathrm{HAsO}_{4}\right)_{2} \mathrm{Mo}_{6} \mathrm{O}_{19}\right]^{6-}$ type anion polyoxomolybdate, have been isolated under the conventional solution method. The crystal structure of the title compound has been elucidated by X-ray crystallography and confirmed by EDS, IR, UV and TG-DTA. There are extensive hydrogen-bonding interactions between the cluster, the cytosinium counter-ions and the water molecules, generating a 3D supramolecular network.

\section{Supplementary material}

CCDC 903129 contains the supplementary crystallographic data for this paper. These data can be obtained free of charge via http://www.ccdc.cam.ac.uk/data_request/cif, by e-mailing data_request@ccdc.cam.ac.uk or by contacting The Cambridge Crystallographic Data Centre, 12 Union Road, Cambridge CB2 1EZ, UK. Fax: +44 1223336033.

\section{Acknowledgements}

The crystal data collection of the compound was done in the 'Laboratoire de Matériaux et Cristallochimie, Faculté des Sciences, El Manar, 2092 Tunis, Tunisia'. We are grateful to Ahmed Driss who supervised this experiment.

\section{References}

1. Pope M T 1983 Heteropoly and isopoly oxometalates (New York: Springer)

2. Katsoulis D E 1998 Chem. Rev. 98359

3. Mizuno N and Misono M 1998 Chem. Rev. 98199

4. Rhule J T, Hill C L and Judd D A 1998 Chem. Rev. 98327
5. Strong J B, Yap G P A, Ostrander R, Liable-Sands L M, Rheingold A L, Thouvenot R, Gouzerh P and Maatta E A 2000 J. Am. Chem. Soc. 122639

6. Pope M T and Müller A 1991 Angew. Chem. Int. Ed. Engl. 3034

7. Pope M T and Müller A 2001 Polyoxometalate chemistry from topology via self-assembly to applications (Dordrecht: Kluwer Academic Publishers)

8. Hagrman P J, Hagrman D and Zubieta J 1999 Angew. Chem. Int. Ed. Engl. 382638

9. Shivaiah V and Das S K 2005 Inorg. Chem. 448846

10. Corma A 1995 Chem. Rev. 95559

11. Heng Z, Newkome G R and Hill C L 2000 Angew. Chem. Int. Ed. Engl. 391771

12. Lin B Z, Chen Y M and Liu P D 2003 Dalton Trans. 122474

13. Haushalter R C and Mundi L A 1992 Chem. Mater. 431

14. Lightfoot P and Masson D 1995 Mater. Res. Bull. 301005

15. Ledain S, Leclaire A, Borel M M, Provost J and Raveau B 1996 J. Solid State Chem. 12424

16. Khan M I, Chen Q and Zubieta J 1993a Inorg. Chem. 322924

17. Khan M I, Chen Q and Zubieta J 1993b J. Chem. Soc. Chem. Commun. 4356

18. Hsu K F and Wang S L 1997 Inorg. Chem. 363049

19. Wang S L, Hsu K F and Nieh Y P 1994 J. Chem. Soc. Dalton Trans. 111681

20. CAD4 1989 Solfware, version 5.0 (Delft, The Netherlands: Enraf Nonius)

21. North A C T, Phillips D C and Mathews F S 1968 Acta Crystallogr. 24351

22. Sheldrick G M 1997a SHELXS-97. Program for the solution of crystal structures (Federal Republic of Germany: University of Göttingen)

23. Sheldrick G M 1997b SHELXL97. Program for the refinement of crystal structures (Federal Republic of Germany: University of Göttingen)

24. Lindqvist I 1953 Ark. Kemi. 5247

25. Strukan N, Cindric M, Devcic M, Giester G and Kamenar B 2000 Acta Crystallogr. C56 278

26. Brown I D and Altermatt D 1985 Acta Crystallogr. Sect B 41 244

27. Yadav R A, Yadav P N S and Yadav J S 1988 Spectrochim. Acta 44A 1201

28. Ma Y, Lu Y, Wang E, Xu X, Guo Y, Bai X and Xu L 2006 J. Mol. Struct. 78418

29. Zhang X M, Shen B Z, You X Z and Fun H K 1997 Polyhedron 1695

30. Yamase T 1998 Chem. Rev. 98307

31. Gong Y, Hu C, Li H, Tang W, Huang K and Hou W $2006 J$. Mol. Struct. $\mathbf{7 8 4} 228$

32. Qi Y, Li Y, Wang E, Jin H, Zhang Z, Wang X and Chang S 2007 Inorg. Chim. Acta 3601841

33. Qi Y, Wang E, Li J and Li Y 2009 J. Solid State Chem. 182 2640

34. Sun C, Li Y, Wang E, Xiao D, An H and Xu L 2007 Inorg. Chem. 461563

35. Kwak W, Rajkovic L M, Stalick J K, Pope M T and Quicksall C O 1976 Inorg. Chem. 152778

36. Brandenburg K 1997 DIAMOND visual crystal structure information system Version 2.1e Crystal Impact GbR, Bonn, Germany 